\title{
Comparison of Functional and Clinical Outcomes between Minimally-Invasive and Conventional Approaches after Total Hip Replacement
}

\author{
Goetz J ${ }^{1 *}$, Renkawitz $\mathbf{T}^{2}$, Meyer $\mathbf{M}^{1}$, Woerner $\mathbf{M}^{3}$, \\ Biedermann $\mathbf{R}^{\mathbf{4}}$, Grifka $\mathrm{J}^{\mathbf{1}}$ and Weber $\mathbf{M}^{\mathbf{1}}$ \\ ${ }^{1}$ Department of Orthopedics, University Hospital of \\ Regensburg, Asklepios Klinikum Bad Abbach, Kaiser- \\ Karl-V.-Allee 3, 93077 Bad Abbach, Germany \\ ${ }^{2}$ Department of Orthopedics and Trauma Surgery, \\ University Hospital of Heidelberg, Schlierbacher \\ Landstraße 200a, 69118 Heidelberg, Germany \\ ${ }^{3}$ Department of Orthopedics, Hospital of Bamberg, Buger \\ Straße 80, 96049 Bamberg, Germany \\ ${ }^{4}$ Department of Orthopedics, University Hospital of \\ Innsbruck, Anichstraße 35, 6020 Innsbruck, Austria \\ *Corresponding author: J ulia Goetz, Department \\ of Orthopedics, University Hospital of Regensburg, \\ Asklepios Klinikum Bad Abbach, Kaiser-Karl-V.-Allee 3, \\ 93077 Bad Abbach, Germany
}

Received: J uly 31, 2021; Accepted: September 01, 2021; Published: September 08, 2021

\begin{abstract}
Background: Total Hip Arthroplasty (THA) is one of the most commonly performed and successful orthopaedic surgeries. At the same time, the issue about the best surgical approach for THA remains controversial. This systematic review aims to evaluate the current evidence for the use of Minimally-Invasive Surgery (MIS) in THA.
\end{abstract}

Methods: A systematic literature search of PubMed, Medline and Embase was conducted. Randomised controlled trials, comparative studies, and cohort studies were included in this systematic review. Main outcome measurements included incision length, blood loss, operating time, length of stay, complications, postoperative pain on a Visual Analogue Scale (VAS), Short Form 36/12 Health Survey (SF 36/12), Harris Hip Score (HHS) and cup inclination.

Results: A total of 30 studies met the inclusion criteria. There was no significant difference between MIS and conventional approaches for THA with regards to complication rates and implant inclination angle. The average operating time in $10 / 24(41 \%)$ studies was significantly $(p<0.05)$ longer in the MIS group. MIS THA lead to an improvement, patient-centered results with reduced blood loss in 9/16 (56\%), reduced use of analgesics in 4/4 (100\%) and reduced myoglobin correlated muscle trauma in $3 / 4(75 \%)$ of the analysed studies. Additionally, 10/10 (100\%) studies reported less postoperative pain after MIS THA, 16/19 (84\%) studies detected an improved postoperative Harris Hip score and $7 / 7$ (100\%) studies an improved SF36/12 score respectively. This resulted a reduced length of stay in 10/10 (100\%) of the studies when compared to THA utilizing a conventional approach.

Conclusion: MIS in THA is nowadays no longer seen as just cosmetically attractive but rather as a real improvement for the clinical outcome. There is evidence for improved patient related outcome following MIS THA.

Keywords: Total Hip Arthroplasty; Harris Hip Score; Visual Analogue Scale

\section{Introduction}

Total Hip Arthroplasty (THA) is considered as one of the most successful treatments for advanced hip osteoarthritis. There is an ongoing debate about the most effective surgical approach for THA [1-3]. Different surgical approaches for THA (Figure 1) are available and the orthopaedic surgeon can choose between traditional and various minimally-invasive THA approaches in different positions [4-9]. The advantages of MIS techniques have been discussed as cosmetic aspects, lower soft tissue/muscle trauma [10-13], lower blood loss [14-18] and reduced postoperative pain symptoms [19-21].

In this context, MIS surgery in THA could lead to faster mobility with shorter hospital stays and faster follow-up treatment, resulting in a cost reduction for the inpatient sector [22] and less downtime for working patients. Critics of MIS THA procedures argue that reduced visibility into the surgical field increases the risk of implant misalignment $[23,24]$. The present paper offers a comprehensive literature analysis with a focus on the most recent data from clinically relevant studies comparing traditional and MIS approaches for THA.

\section{Minimally invasive "Super Path" approach}

The Super Path approach technique was first described by Chow et al. [25] in 2011. In a lateral decubitus position, with the operated leg in approximately $60^{\circ}$ of flexion, and 20 to $30^{\circ}$ of internal rotation in maximal adduction, the skin incision is made between the tips of the greater trochanter in line with the femoral axis. The approach uses the space between the piriformis posterior and the anterior gluteus minimus/medius muscle.

\section{Minimally invasive direct anterior (DA) approach}

The minimally-invasive Direct Anterior (DA) approach, which was initially described in 1870 by Hueter [26] and subsequently by Smith-Petersen et al. [27] and Judet et al. [28]. It is an intermuscular and internervous approach. The patient is in supine position. Both legs are flexibly covered. This makes it possible to adduct the leg during the operation and to cross it in an extended position. First, the skin is incised distal and lateral to the Anterior Superior Iliac Spine (ASIS) 


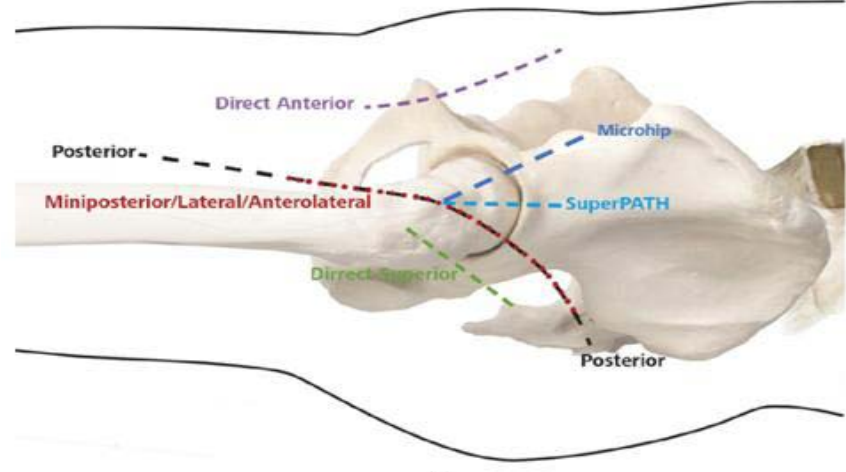

A)

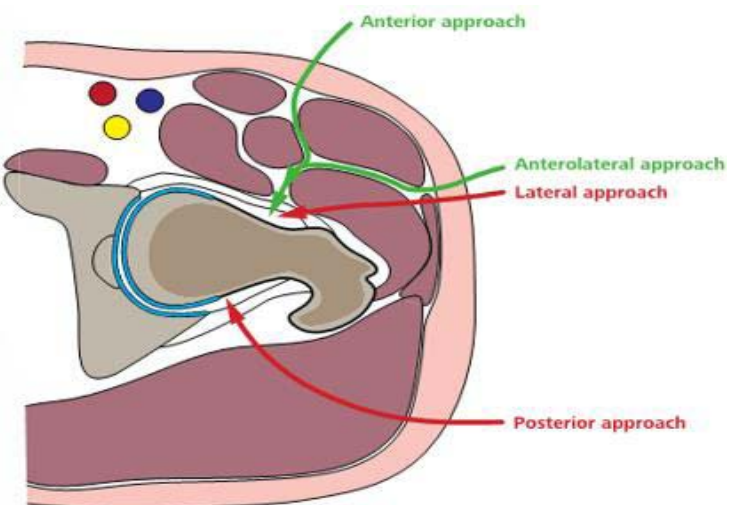

B)

Figure 1: Graphical representation of the commonly used incisions for THA. A) Skin incisions B) Preparation/incisions of the muscle groups.
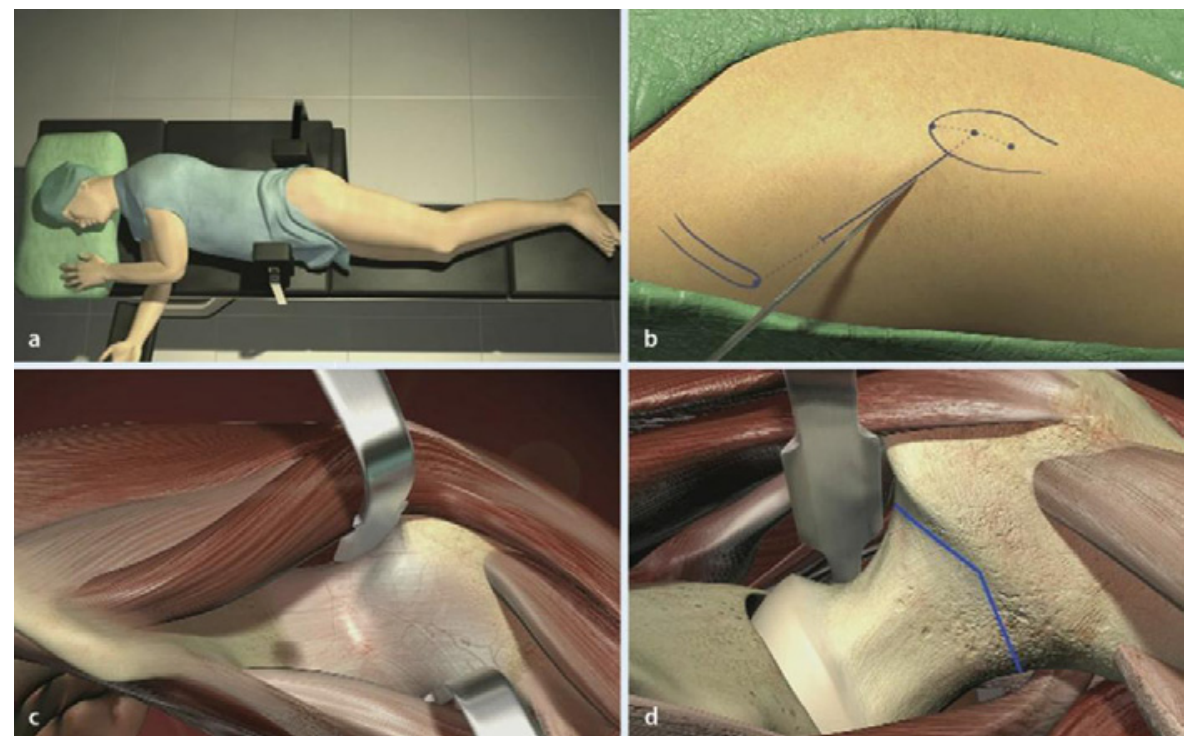

Figure 2: Example of anterolateral MIS THA. A) The patient is in a lateral position and the surgeon is ventral to the patient. The dorsal foot section of the operating table is removed in order that the patient leg can be moved intraoperatively dorsal in a hyperextended position. This is important to represent the femur B) On the imaginary line connecting the anterior superior iliac spine and the anterior edge of the center of the trochanter massive, the skin incision is made. C) Following the incision of the tractus fascia, the muscle gap between the tensor fasciae latae and the rectus or sartorius will be palpated. A blunt preparation is performed without detachment of the muscle groups. D) The joint capsule, shown in the picture, is incised. After the underlying femoral neck will be osteotomized with a stepped incision. By courtesy of the company DePuy Orthopaedics, Kirkel, Germany.

to a point a few fingerbreadths anterior to the greater trochanter. If necessary, it can then be extended proximally and distally.

\section{Minimally invasive anterolateral (Microhip) approach}

The MicroHip approach was initially described by Michel and Witschger [29]. No detachment of tendons or splitting of muscles is needed. As shown in Figure 2 the patient is in a lateral decubitus position and the surgeon is ventral to the patient. Various authors have pointed out that hyperextension of the leg is necessary for a true muscle considering procedure, which can only be obtained in a lateral decubitus position [31]. The skin incision is made on the imaginary line connecting the anterior superior iliac spina and the anterior edge of the center of the trochanter massive. Following the incision of the tractus fascia, the muscle gap between the tensor fasciae latae and the rectus or sartorius will be palpated. With preparation of anterolateral muscular interval the exposure and incision of capsule is possible. A blunt preparation is performed without detachment of the muscle groups. The joint capsule then incised. A leg positioning in external rotation and hyperextension is necessary for neck osteotomy and femoral preparation.

\section{Direct lateral approach}

Although several versions of direct lateral approach for THA have been used since McFarland and Osborne (1954) [32] the modern direct lateral approach was described by Hardinge [33] and also subsequently by Bauer et al. [34]. The Patient is in supine position with the advantage of a good interoperative view but the risk to injure the superior gluteal nerve. Normally the incision is proximal to the greater trochanter and runs along the femoral shaft. The fascia is then incised longitudinally. After identifying the anterior and posterior 
border of gluteus medius, the blunt dissection is used to split the muscle in line with its fibers. Vastus lateralis should be exposed next and split longitudinally. To allow the surgeon visualization of femoral neck the leg is placed in extreme adduction and external rotation.

\section{Posterior approach}

The posterior approach for THA was described separately by Moore (1952) [35] Kocher (1911) [36] and von Langenbeck (1874) [37]. The patient is placed in the lateral position and the incision is made from the posterior iliac spine to the greater trochanter. The fascia lata and iliotibial band are incised longitudinally and proximally to split along the fibers of gluteus maximus. It provides direct access to the outer surface of the posterior column. This is accomplished through the splitting of the muscle fibers of the gluteus maximus and the release of its tendinous femoral insertion along with the release of the piriformis. It has the benefit of not interfering with the abductor mechanism, however, there is a risk of damaging the sciatic nerve. Although some soft tissue preparation is needed, some authors like Fink et al. or Schmidt-Breakling et al. have published a "minimally invasive" adaptions of the posterior approach $[38,39]$.

\section{Methods}

A literature search of PubMed, Medline and Embase was conducted. The search criteria were hip arthroplasty(ies)/ replacement(s), minimally invasive/MIS/mini incision, and/or approach/anterior approach/direct anterior/Microhip/Super Path Randomised controlled trials, comparative studies, and cohort studies were included. A total of 1043 studies were found which compared MIS THA and THA performed through a standard approach (Figure 3). Cadavers and surface replacement studies, studies with hemi-prostheses or reconstructions after fracture were excluded. Studies using multiple incisions or using an orthopaedic navigation system were also excluded. Studies between 2008-2019 and with a minimum of two outcome criteria were included. The outcomes criteria were: incision length, blood loss, operating time, length of stay, complications, implant position, pain on a Visual Analogue Scale (VAS), Short Form (SF) 36/12, Harris Hip Score (HHS) and laboratory parameters associated with muscular trauma (creatine kinase (CK) and myoglobin concentration). The operating time is measured between incision and suture. In accordance with the defined quality criteria, 30 studies were selected for further analysis. These 30 studies are shown in Table 1.

\section{Statistical analysis}

In order to compare studies, effect sizes, $\mathrm{p}$-values and mean values are indicated. The latter were plotted as charts and tables. To ensure comparability, the $\mathrm{p}$-values were given with a significance level of $\mathrm{p}$ $\leq 0.05(5 \%)$.

\section{Results}

Table 2 summarises all results of the included studies. To ensure comparability, the $\mathrm{p}$-values were given with a significance level of $\mathrm{p}$ $\leq 0.05$ (5\%). Improved patient-centered results for MIS THA in Table 2 are marked with a green field, improved patient-cantered results for standard THA are marked with a red field.

\section{Incision}

A number of authors compared the incision length of MIS approaches and the standard approaches for THA. In summary, $10 / 11(90 \%)$ of the studies found a shorter incision length in the MIS groups (Table 2). A significant $(\mathrm{p}<0.05)$ difference was described in three papers. We did not find any data comparing the lengths of the incisions between the anterolateral and direct anterior approach.
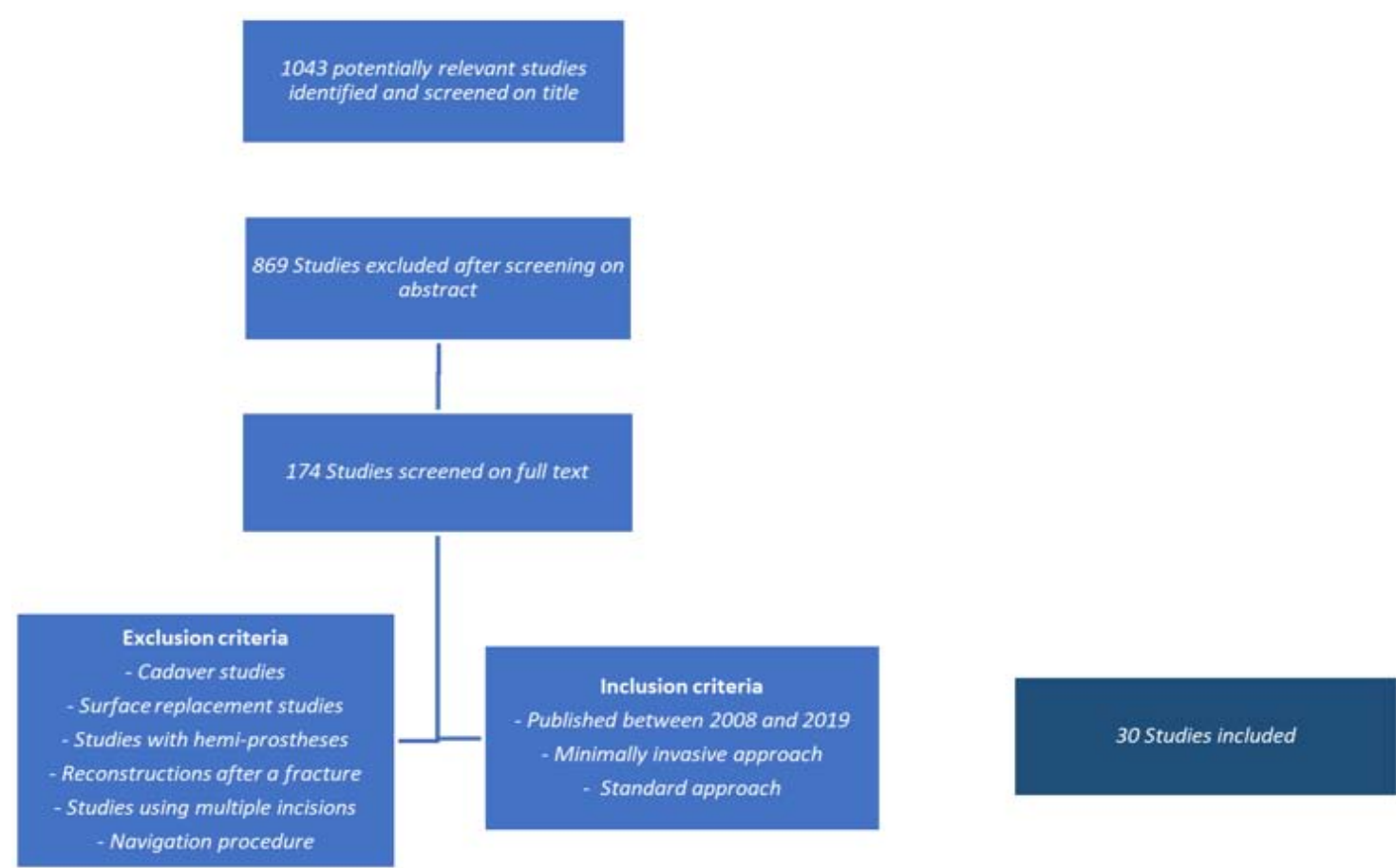

Figure 3: Flowchart showing the selection of articles. The following combined key words were used: hip arthroplasty(ies)/replacement(s), minimally invasive/MIS/ mini incision, and/or approach/anterior approach/direct anterior/Microhip/Super Path. 
Table 1: Total Hip replacement with minimally invasive approaches versus conventional posterior approaches. A total of 30 studies met the inclusion criteria.

\begin{tabular}{|c|c|c|c|c|c|c|}
\hline Authors & Publication Years & Study Design & $\begin{array}{c}\text { Study } \\
\text { Participants }\end{array}$ & $\begin{array}{c}\text { minimally Invasive } \\
\text { Approach }\end{array}$ & Standard Approach & Follow-up \\
\hline Alecci et al. & 2011 & Retrospective & 221/198 & MI direct anterior & Lateral & Hospital stay \\
\hline $\begin{array}{l}\text { Bala Subramaniam } \\
\text { et al. }\end{array}$ & 2016 & Retrospective & $50 / 42$ & Anterior & Posterior & 1 year \\
\hline Brismar et al. & 2018 & $\begin{array}{l}\text { Randomized parallel } \\
\text { group }\end{array}$ & $50 / 50$ & MI direct anterior & Direct Lateral & 5 years \\
\hline D'Arrigo et al. & 2009 & PRC & $60 / 149$ & $\begin{array}{l}\text { MI Lateral/anterior/ } \\
\text { anterolateral }\end{array}$ & Standard Lateral & 6 weeks \\
\hline Dienstknecht T et al. & 2014 & PRC & $55 / 88$ & Micro-hip & Transgluteal (Bauer) & 6 days \\
\hline Fink et al. & 2010 & PC & $50 / 50$ & MI Posterior & Posterior & 6 weeks \\
\hline Goosen et al. & 2011 & PRC & $60 / 60$ & Anterolateral & Posterolateral & 1 years \\
\hline Hozack et al. & 2008 & PRC & $43 / 36$ & Direct anterior & Direct Posterior & 6 months \\
\hline Ilchmann et al. & 2013 & Consecutive Cohort & $113 / 142$ & MI anterior & Lateral & 2 years \\
\hline Kawarai et al. & 2017 & Retrospective & $106 / 109$ & Direct anterior & Anterolateral & 1 year \\
\hline Martin et al. & 2011 & PRC & $42 / 41$ & Anterolateral (Rottinger Almis) & Transgluteal - Hardinge & 1 year \\
\hline Mas Martinez et al. & 2009 & PRC & $30 / 60$ & Super Path & Posterior & 1 year \\
\hline Matziolis et al. & 2011 & PRC & $20 / 20$ & Anterolateral & Transgluteal & 1 year \\
\hline Mazoochian et al. & 2009 & PRC & $26 / 26$ & Modified Hardinge & lateral & 3 months \\
\hline Mouilhade et al. & 2011 & PC & $92 / 49$ & $\begin{array}{c}\text { Mini-Watson-Jones or } \\
\text { Rottinger }\end{array}$ & Anterolateral transgluteal & 6 months \\
\hline Muller et al. & 2011 & PRC & $21 / 16$ & Anterolateral & Direct Lateral & 1 year \\
\hline Ouyang et al. & 2018 & PRC & $12 / 12$ & Super Path & Posterolateral & 1 year \\
\hline Pogliacomi et al. & 2012 & PC & $35 / 35$ & Anterior & Lateral & 1 year \\
\hline Reichert et al. & 2018 & PRC & $77 / 71$ & MI direct anterior & $\begin{array}{c}\text { Direct Transgluteal } \\
\text { Lateral }\end{array}$ & 1 year \\
\hline Rykov et al. & 2017 & PRC & $23 / 23$ & Direct anterior & Posterolateral & 6 weeks \\
\hline Sendter et al. & 2011 & PRC & $74 / 60$ & Micro-hip & Transglutealen (Bauer) & 1 year \\
\hline Sirtori et al. & 2018 & Prospective Longitudinal & $12 / 12$ & MI direct anterior & Postero-lateral & 90 days \\
\hline $\begin{array}{l}\text { Varela Egocheaga } \\
\text { et al. }\end{array}$ & 2010 & PRC & $25 / 25$ & Posterior MIS & Posterior Standard & 1 year \\
\hline Wayne et al. & 2009 & PC & $100 / 100$ & MI anterior & Lateral - Hardinge & Hospital stay \\
\hline Wohlrab et al. & 2008 & PRC & $20 / 20$ & Modified Waston-Jones & Bauer & 3 months \\
\hline Xie et al. & 2017 & PRC & $46 / 46$ & Super Path & Posterior & 1 year \\
\hline Yan et al. & 2017 & PC & $70 / 103$ & Super Path & Lateral (Hardinge) & 15 months \\
\hline Yang et al. & 2010 & PRC blind & $55 / 55$ & Modified Anterolateral & Posterolateral & 3 years \\
\hline Yuan et al. & 2018 & PRC & $40 / 30$ & Super Path & Posterolateral & 6-18 months \\
\hline Zhao et al. & 2017 & PC & $60 / 92$ & Direct anterior & Posterolateral & $2-2.5$ years \\
\hline
\end{tabular}

Abbreviations: PRC: Prospectively Randomized Controlled; PC: Prospectively Controlled; MI: Minimally Invasive [40-69].

\section{Operating time}

The operating time (incision-suture time) was recorded in 28 studies and the consensus was that MIS THA took longer than conventional approaches. Figure 4 shows the mean operating times for the different approaches. In four publications, the operating time was not described in detail. All in all, 10/24 (41\%) of the studies reported a significant $(\mathrm{p}<0.05)$ shorter operating time for standard approaches.

\section{Blood loss}

Blood loss was recorded with intraoperative measurements of blood loss in milliliters. Less blood loss for the MIS approaches were described in 9/16 (56\%) studies.

\section{Creatine kinase and myoglobulin concentration}

The Creatine kinase concentration was measured in a total of 7/30
(23\%) studies. Ouyang et al. reported a significantly lower Creatine kinase concentration through the Super Path approach. Furthermore, $6 / 30(20 \%)$ studies measured the concentration of myoglobin in the blood.

\section{Analgesics and visual analogue scale (VAS)}

In 4/4 (100\%) studies, patients needed less analgesics after MIS THA. This was also reflected in the evaluation of the visual analogue scale. Overall, $7 / 10(70 \%)$ studies noticed significantly lower postoperative pain on a visual analogue scale after MIS THA.

\section{Trendelenburg sign}

The incidence of the trendelenburg sign (as a correlate for weak or paralyzed abductor muscles of the hip) was evaluated in the context of two study protocols. No difference between MIS and conventional THA was reported in the study by Sendtner et al. [73] and Mueller et 
Table 2: To ensure comparability, the $p$-values were given with a significance level of $p \leq 0.05$ ( $5 \%)$. The minimally invasive approaches showed better results in the green marked fields and the standard approaches in the red fields.

\begin{tabular}{|c|c|c|c|c|c|c|c|c|c|c|c|c|c|}
\hline Authors & $\begin{array}{c}\text { Incision } \\
{[\mathrm{cm}]}\end{array}$ & $\begin{array}{c}\text { Operation time } \\
{[\mathrm{min}]}\end{array}$ & Blood loss [ml] & $\begin{array}{c}\text { Creatine } \\
\text { kinase }\end{array}$ & $\begin{array}{c}\text { Myoglobin } \\
\text { concentration }\end{array}$ & Analgesics & VAS & $\begin{array}{c}\text { Trendelenburg } \\
\text { sign }\end{array}$ & $\begin{array}{c}\text { HHS } \\
\text { postop }\end{array}$ & $\begin{array}{l}\text { SF } 36 / \\
\text { SF } 12\end{array}$ & $\begin{array}{l}\text { Hospital } \\
\text { stay }\end{array}$ & $\begin{array}{l}\text { Postoperative } \\
\text { complications }\end{array}$ & $\begin{array}{c}\text { Implant } \\
\text { inclination }\end{array}$ \\
\hline Alecci et al. & - & $89 / 81$ & $\mathrm{Ml}$ anterior $\mathrm{P}<0.05$ & - & 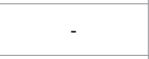 & - & $\begin{array}{c}\text { MI anterior } \\
<\text { Lateral }\end{array}$ & - & - & - & $7 / 10$ & r $=0=2$ & - \\
\hline $\begin{array}{l}\text { Bala } \\
\text { subramaniam } \\
\text { et al. }\end{array}$ & - & $\begin{array}{l}83 / 91.8 \\
\mathrm{P}=0.048\end{array}$ & - & - & - & - & - & - & $\begin{array}{l}87.5 / 78.0 \\
P=0.051\end{array}$ & $\begin{array}{c}\text { Anterior > } \\
\text { posterior } \\
\mathrm{P}=0.061\end{array}$ & $\begin{array}{l}4.2 / 6.0 \\
P=0.004\end{array}$ & $P>0.05$ & - \\
\hline Brismar et al. & - & $101 / 80 P<0.05$ & $325 / 300 \mathrm{P}>0.05$ & - & - & $\begin{array}{l}\mathrm{DA}<\mathrm{DL} \\
\mathrm{P}>0.05\end{array}$ & DA $P<0.05$ & - & $\mathrm{DA} P<0.05$ & - & $\begin{array}{c}\mathrm{DA} \\
\mathrm{P}=0.006\end{array}$ & $7 / 0 P>0.05$ & - \\
\hline D'Arrigo et al. & - & $\begin{array}{c}\text { 102/121/110/77 } \\
\text { (lateral vs. } \\
\text { MI anterior } \\
\mathrm{P}=0.013 \text { ) }\end{array}$ & $\begin{array}{c}\text { MI lateral } \\
\mathrm{P}=0.002 / \\
\text { anterior } \mathrm{P}=0.004 / \\
\text { anterolateral } \\
\mathrm{P}=0.007\end{array}$ & - & - & - & - & - & $P>0.05$ & - & $P>0.05$ & $\begin{array}{c}\text { Standard lateral } \\
\quad P=0.003\end{array}$ & $P>0.05$ \\
\hline $\begin{array}{l}\text { Dienstknecht } \\
\text { T et al. }\end{array}$ & $\begin{array}{l}9.3 / 13.4 \\
P<0.01\end{array}$ & $60 / 68 \mathrm{P}=0.021$ & Micro-hip $\mathrm{P}<0.001$ & - & - & - & $\begin{array}{l}\text { Micro-hip } \\
\text { P }<0.05\end{array}$ & - & - & - & - & $P>0.05$ & - \\
\hline Fink et al. & - & - & MIS $>P$ & $P>0.05$ & $P>0.05$ & $\mathrm{MIS}<\mathrm{P}$ & - & - & - & - & - & $P>0.05$ & $P>0.05$ \\
\hline Goosen et al. & $7.8 / 18$ & $\mathrm{AL}<\mathrm{PL}$ & $P>0.05$ & $P>0.05$ & $P>0.05$ & - & - & - & $77 / 72$ & - & - & $P>0.05$ & $47^{\circ} / 47^{\circ}$ \\
\hline Hozack et al. & $\begin{array}{c}9-12 / 8- \\
12 \\
P>0.05\end{array}$ & $P>0.05$ & $P>0.05$ & - & - & - & $P>0.05$ & - & $\begin{array}{c}94.6 / 88.6 \\
P>0.05\end{array}$ & $\begin{array}{c}\text { Direct } \\
\text { anterior } \\
\mathrm{P}<0.05\end{array}$ & $P>0.05$ & $P>0.05$ & - \\
\hline $\begin{array}{l}\text { Illchmann } \\
\text { et al. }\end{array}$ & - & $\begin{array}{l}119 / 107 \\
P<0.001\end{array}$ & $700 / 700 \mathrm{P}=0.291$ & - & - & - & $\begin{array}{c}\text { MI anterior } \\
\mathrm{P}<0.05\end{array}$ & - & MIS $P<0.05$ & - & $\begin{array}{c}11 / 12 \\
P<0.001\end{array}$ & $P>0.05$ & $P>0.05$ \\
\hline Kawarai et al. & - & - & - & - & - & - & - & - & - & - & - & $5 \% / 2 \% P=0.275$ & $\begin{array}{c}42.5^{\circ} / 42.5^{\circ} \\
P=0.927\end{array}$ \\
\hline Martin et al. & - & $\begin{array}{c}\mathrm{AL}> \\
\text { Transgluteal }\end{array}$ & AL $>$ Transgluteal & - & - & $P>0.05$ & - & - & $P>0.05$ & $P>0.05$ & $P>0.05$ & $P>0.05$ & $P>0.05$ \\
\hline $\begin{array}{l}\text { Mas Martinez } \\
\text { et al. }\end{array}$ & - & $\begin{array}{c}\text { Posterior } \\
P<0.05\end{array}$ & Posterior $\mathrm{P}<0.05$ & - & - & - & - & - & & $\begin{array}{c}\text { Super Path } \\
>\mathrm{P}\end{array}$ & $P>0.05$ & - & $P>0.05$ \\
\hline $\begin{array}{l}\text { Matziolis } \\
\text { et al. }\end{array}$ & - & - & - & - & - & - & - & - & $84 / 86$ & - & - & - & $45.9^{\circ} / 45.5^{\circ}$ \\
\hline $\begin{array}{l}\text { Mazoochlan } \\
\text { et al. }\end{array}$ & $8.9 / 14$ & $77 / 91$ & $502 / 660$ & $P>0.05$ & MIS $<$ L & & & & $87 / 78$ & & & $P>0.05$ & $46.3^{\circ} / 45.3^{\circ}$ \\
\hline $\begin{array}{l}\text { Moullhade } \\
\text { et al. }\end{array}$ & - & 93/71 $\mathrm{P}<0.001$ & $P>0.05$ & $\begin{array}{l}385 / 479 \\
P=0.04\end{array}$ & $\begin{array}{c}285.7 / 266 \\
P=0.68\end{array}$ & $\begin{array}{c}\text { W.J, } \\
\text { Transgluteal } \\
\mathrm{P}<0.001\end{array}$ & - & - & $\begin{array}{l}88.2 / 76 \\
P<0.001\end{array}$ & $\begin{array}{c}43.2 / 39.6 \\
P>0.05\end{array}$ & $\begin{array}{c}9 / 10 \\
P>0.05\end{array}$ & $P>0.05$ & $\begin{array}{l}46^{\circ} / 47^{\circ} \\
P>0.05\end{array}$ \\
\hline Muller et al. & $8 / 10.4$ & $51 / 50$ & - & - & MIS $<$ L & - & - & $1 / 6$ & $80 / 76$ & - & - & - & - \\
\hline Ouyang et al. & $\begin{array}{c}\text { Super } \\
\text { Path } \\
P=0.041\end{array}$ & $\begin{array}{l}\text { Posterolateral } \\
\mathrm{P}<0.001\end{array}$ & $P>0.05$ & $\begin{array}{c}\text { Super Path } \\
P<0.05\end{array}$ & - & - & $\begin{array}{c}\text { Super } \\
\text { Path } \\
P<0.05\end{array}$ & - & $\begin{array}{l}\text { Super Path } \\
P<0.05\end{array}$ & - & $P=0.640$ & $1 / 0 P>0.05$ & $P>0.05$ \\
\hline $\begin{array}{l}\text { Pogliacomi } \\
\text { et al. }\end{array}$ & 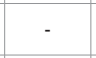 & $93 / 90$ & $\begin{array}{c}600.1 / 629.4 \\
P>0.05\end{array}$ & - & - & - & $P<0.05$ & - & $\begin{array}{c}91.39 / 91.51 \\
P>0.05\end{array}$ & - & - & - & $\begin{array}{c}46.1^{\circ} / 46.8^{\circ} \\
P>0.05\end{array}$ \\
\hline Reichert et al. & & & & & & & $P>0.05$ & & $\begin{array}{c}92.4 / 91.43 \\
P=0.477\end{array}$ & $\begin{array}{l}\text { Anterior } \\
\mathrm{P}=0.017\end{array}$ & & $P>0.05$ & $\begin{array}{c}38.6 / 40.3^{\circ} \\
P>0.05\end{array}$ \\
\hline Rykov et al. & & $\begin{array}{c}\text { Posterolateral } \\
\mathrm{P}=0.001\end{array}$ & & $P>0.05$ & & & & & $P>0.05$ & & & & \\
\hline Sendter et al. & $\begin{array}{l}8.5 / 15.5 \\
P<0.05\end{array}$ & $77 / 69 P<0.05$ & $\mathrm{MH} P<0.05$ & & & $P<0.05$ & & $P<0.05$ & $\begin{array}{c}92 / 92 \\
P>0.05\end{array}$ & & & $3 / 0 P>0.05$ & $\begin{array}{l}47^{\circ} / 49^{\circ} \\
P>0.05\end{array}$ \\
\hline Sirtori et al. & & $P>0.05$ & DAA $P<0.05$ & & & & $\begin{array}{l}\text { Mini-DAA } \\
P<0.05\end{array}$ & & $\mathrm{DAA}>\mathrm{PL}$ & DAA $>$ PL & & & \\
\hline $\begin{array}{l}\text { Varela } \\
\text { Egocheaga } \\
\text { et al. }\end{array}$ & & $P>0.05$ & $P>0.05$ & & & MIS $<$ P & & & & & MIS $<$ P & $P>0.05$ & $P>0.05$ \\
\hline Wayne et al. & & $115 / 98 P<0.05$ & $\mathrm{MI}$ anterior $\mathrm{P}<0.05$ & & & & & & & & $\begin{array}{c}\text { MI P }<0.05 \\
\text { hospital } \\
\text { stay }\end{array}$ & $\mathrm{x} 1$ & $P>0.05$ \\
\hline Wohlrab et al. & & $57 / 61$ & & $P>0.05$ & MIS $<$ L & & $P>0.05$ & & $96 / 91$ & & & $P>0.05$ & $48.3^{\circ} / 48.9^{\circ}$ \\
\hline Xie et al. & $\begin{array}{l}\text { Super } \\
\text { Path } \\
P<0.05\end{array}$ & $P>0.05$ & $P>0.05$ & & & & $\begin{array}{l}\text { Super } \\
\text { Path } \\
P<0.05\end{array}$ & & $\begin{array}{l}\text { Super Path } \\
P<0.05\end{array}$ & & $\begin{array}{c}\text { Super Path } \\
P<0.05\end{array}$ & $P>0.05$ & $P>0.05$ \\
\hline Yan et al. & $\begin{array}{c}\text { Super } \\
\text { Path } \\
\mathrm{P}<0.05\end{array}$ & $\begin{array}{c}\text { Lateral } \\
\text { (Hardinge) } \\
\mathrm{P}<0.05\end{array}$ & $\begin{array}{c}\text { Lateral (Hardinge) } \\
\mathrm{P}<0.05\end{array}$ & & & & $\begin{array}{c}\text { Super } \\
\text { Path } \\
\mathrm{P}<0.05\end{array}$ & & $\begin{array}{c}\text { Super Path } \\
\mathrm{P}<0.05\end{array}$ & & $\begin{array}{c}\text { Super Path } \\
\mathrm{P}<0.05\end{array}$ & $P>0.05$ & \\
\hline Yang et al. & $\begin{array}{l}7.5 / 15.2 \\
P<0.01\end{array}$ & $78 / 74 P>0.05$ & $376 / 605 P<0.01$ & & & & $\begin{array}{c}\text { Modified } \\
\text { AL } P<0.01\end{array}$ & & $\begin{array}{c}83.80 / 74.96 \\
P<0.01\end{array}$ & & & & $\begin{array}{c}48.3^{\circ} / 48.9^{\circ} \\
P>0.05\end{array}$ \\
\hline Yuan et al. & $\begin{array}{l}\text { Super } \\
\text { Path } \\
P<0.05\end{array}$ & $\begin{array}{l}\text { Super Path } \\
\mathrm{P}<0.05\end{array}$ & $\begin{array}{l}\text { Super Path } \\
\mathrm{P}<0.05\end{array}$ & & & & & & $P>0.05$ & $\begin{array}{c}\text { Super Path } \\
\mathrm{P}<0.05\end{array}$ & & & $P>0.05$ \\
\hline Zhao et al. & & $\begin{array}{c}\text { Posterolateral } \\
P<0.05\end{array}$ & $\begin{array}{c}\text { Posterolateral } \\
P<0.05\end{array}$ & & & & $\begin{array}{c}\text { DAA } \\
P<0.05\end{array}$ & & DAA $P<0.05$ & & $\begin{array}{c}\text { DAA Group } \\
P<0.05\end{array}$ & $P>0.05$ & $P>0.05$ \\
\hline
\end{tabular}

Abbreviations: MI: Minimal Invasive Approach; AL: Anterolateral Approach; DAA: Direct Anterior Approach; L: Lateral Approach; P: Posterior Approach; p: p-values Significance Level; X1: MI: Significant $(p<0.05)$ increase in intraoperative femur fracture, significantly $(p<0.05)$ fewer infections but higher rate of nerve damage and higher percentage of acetabular component malposition.

al. [70] pointed out that one patient after MIS THA and six patients with the conventional lateral approach had a positive trendelenburg sign.

\section{Harris hip score}

All in all a significant $(\mathrm{p}<0.05)$ difference in the postoperative
HHS was described in 8/23 (35\%) papers. One study found a similar HHS one-year post surgery. It should be noted that the studies covered different study periods. One study reported 5 years, one 3 years, two 2 years, one 15 months, ten 1 year, three 6 months, three 3 months and two 6 weeks follow up. The mean Harris Hip Score 


\section{Operating time $(\mathrm{min})$}

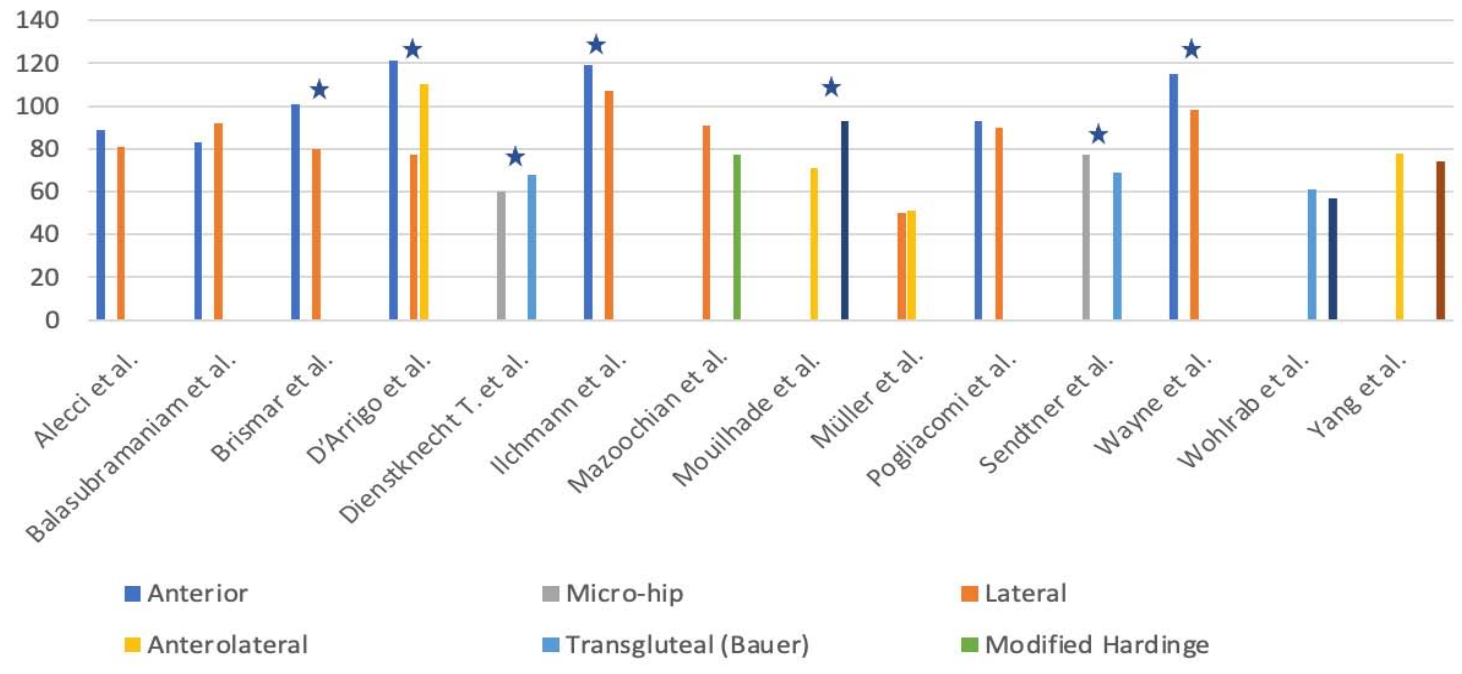

- Mini-Watson-Jones or Rott inger $\mathbf{a}$ Posterolateral

Figure 4: The mean value operating time was recorded in 14 studies and the consensus was that the MIS THA took longer than either the lateral, anterolateral or posterior approaches. All significant $(p<0.05)$ results are marked with an asterisk.

\section{Harris Hip Score}
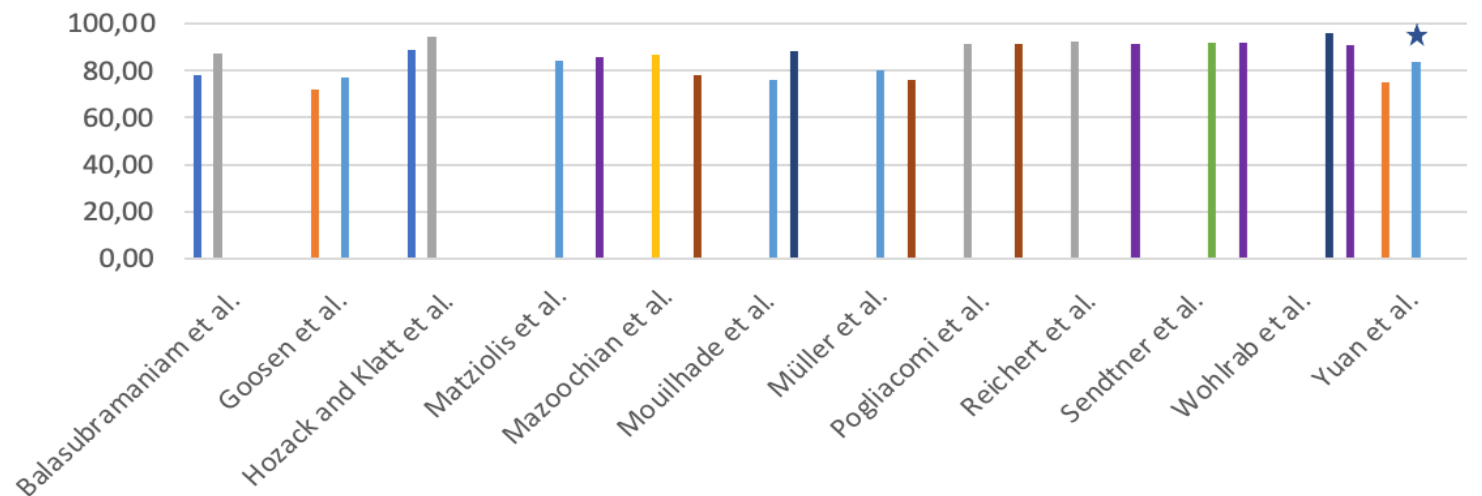

$$
\begin{array}{lll}
\square \text { Posterior } & \text { Posterolateral } & \text { Anterior } \\
\square \text { Modified Hardinge } & \text { Anterolateral } & \text { Micro Hip } \\
\square \text { Mini-Watson-Jones or Rottinger } & \square \text { Lateral } & \text { Transgluteal (Bauer) }
\end{array}
$$

Figure 5: The mean value Harris Hip Score was recorded in 12 studies. The consensus was that patients after MIS THA had a higher mean value Harris Hip Score, in $9 / 12(75 \%)$ studies. All significant $(p<0.05)$ results are marked with an asterisk.

(HHS) was published in 12 of the 23 publications, as you can see in Figure 5.

\section{Short Form Health - SF 36 and SF 12}

The SF36 or SF12 was analysed in 8 study groups. Overall, 7/7 (100\%) of the analysed studies reported an improved health-related quality of life for MIS THA when compared to standard THA. In one publication, it was not described in detail which approach performed better in mean value.

\section{Hospital stays}

The length of stay was analysed in a total of $15 / 30$ (50\%) studies.
In 10/15 (67\%) studies a shorter hospital stay was reported for MIS THA when compared to the conventional approach. In five out of 15 publications detailed information in terms of mean value were missing.

\section{Postoperative complications}

All in all, 20/21 (95\%) studies reported similar post-operative complications (rate of nerve damage, intraoperative femur fracture or infection rate) between THAs undertaken using the MIS THA and conventional approaches. Only D'Arrigo et al. described significant $(\mathrm{p}<0.05)$ lower complications with a standard lateral approach. 


\section{Implant inclination}

Table 2 shows the differences in the inclination angle of the acetabular cups. In summary, 21/21 (100\%) studies reported no significance difference between the post-operative inclination of the acetabular cup between MIS and standard THA.

\section{Discussion}

Our comprehensive literature analysis with a focus on the most recent data from clinically relevant studies comparing traditional and MIS approaches for THA indicate that a less traumatic hip approach results in reduced immediate postoperative pain, better hip function and higher quality of life in the early postoperative period.

Some studies in our analysis could not clearly show how the course of the operation continued after the MIS THA skin incision. Whether the surgical technique was similar in all study, protocols or which steps were different to the conventional one could not be evaluated. Some studies explicitly pointed out that no special instruments were required [71]. There is a broad consensus amongst orthopaedic surgeons, that the main focus of MIS THA is not primarily a smaller skin incision but rather a maximum soft tissue protection without unnecessary detachment of muscle and tendon attachments. The Literature experience with several thousand MIS THA (via a MicroHip $\odot$ approach) confirms that angled milling and impact instruments are indispensable during cup preparation in order to avoid soft tissue damage and cosmetically unsatisfactory scar formation [72-74].

When analyzing the current evidence for MIS THA, we found advantages such as reduced risk of dislocation, faster recovery and less pain. Disadvantages of any MIS THA approach include the learning curve and sometimes a cumbersome positioning of the patient during surgery. Furthermore, MIS THA may be difficult to use on obese and pre-operated patients. Some studies from the beginning of MIS THA report an increased operation time. Newer studies could not confirm this observation. It seems that the longer time of surgery for the MIS THA approach may be attributed to the learning curve in different studies.

The rate of perioperative complications for minimally invasive surgery varies between zero and 26\% [75]. Mjaaland et al. [76] and Kawarai et al. [77] described an unexpectedly high number of complications occurred in the DA group, presumably related to a more demanding surgical technique. Special care should be therefore taken for patients undergoing MIS THA in a supine position. Goulding et al. [78] defined the incidence of lateral femoral cutaneous nerve neuropraxia as a frequent complication after anterior approach THA.

In addition to the lower blood loss, studies of recent years confirmed faster rehabilitation after MIS THA. The advantages regarding the rehabilitation phase are concentrated in the first postoperative months. From the 12th postoperative week onwards, no superiority for the MIS THA patient group can be detected. In summary, it seems that the hospital stays and/or the duration of the convalescence and therefore socio-economic costs for the health system can be reduced by MIS THA procedures predominantly during the first postoperative months [79-81]. On the other hand, the literature search of Llody et al. indicate, "There is insufficient evidence to indicate that surgical technique alone makes a significant difference to recovery" [83]. Important is, as Kehlet et al. emphasized, "first better, then faster" [82].

Most of the randomized trial study results may have been affected by bias since patients and caregivers could not be blinded. Additionally, the location of the scar revealed the approach. Many of these included studies were published at a decade were the method was not widespread, possibly reducing the risk of different expectations linked to either method. Patients were treated by the same postoperative protocol and the results were recorded by a physiotherapist not involved in the recruitment of patients. It is possible that the functional tests used were not demanding enough to detect differences between groups at later time points. Luna et al. accentuate patient reported outcomes should not be used as the only measure of recovery after THA because early improvement in patient-reported physical function (HOOS) does not correlate with objectively assessed function. Due to the missing information in some studies, it was unfortunately not possible to correlate with objectively assessed function [84].

Only few studies considered possible confounding factors that could play a major role in comparative studies. For example, analgesic, anaesthetic the pre-operative level of haemoglobin and medical comorbidities affect the interoperative loss of blood etc. Further studies should account for these effects.

\section{Expert Opinion}

The question is: What are the clearly measurable advantages of minimally invasive surgery in total hip arthroplasty? Naturally, any literature search must be interpreted with caution as MIS and conventional groups differed in many important ways such as BMI or operator experience between the different studies. However, according to the current data and our own experience MIS THA enables a faster return to postoperative functioning due to reduced tissue trauma, lower blood loss and less pain after surgery. These advantages with regard to the rehabilitation phase are concentrated in the first postoperative months. From a socio-economic perspective, shorter hospital stay and a quicker convalescence have the potential to reduce costs for the health care system. Still too often, the term "minimally invasive" is still only associated with a smaller incision length and various invasive, transmuscular approaches (such as the lateral or posterior approach) have been labelled as "MIS", "Micro" or "Mini" only be reducing the incision length but without avoiding detaching the tendon insertions of the stabilizing musculature around the hip joint. However, a true muscle-sparing hip pathway is made through the natural planes between muscle bodies until the joint capsule is reached. From a biomechanical perspective, the preservation of the abductor muscles and the preservation of proprioceptive functioning of the hip aid early rehabilitation. MIS THA may not necessarily be suited for orthopaedic surgeons who perform the procedure only occasionally. The learning curve is long and especially the added technical difficulty caused by reduced visualization may cause higher complication rates in less experienced hands. THA in obese patients or patients with a very stiff musculature can make a minimally invasive approach technically more difficult. Especially in these patients, there is a greater risk of traumatizing the wound edges with retractors. At the same time, clinical data and our own experience demonstrate that MIS THA is a safe procedure in 
high volume centers for hip surgery.

A final judgement about the advantages of MIS THA is limited by the fact that many of the analysed studies did not consider possible confounding factors that could play a major role in comparative studies. In the end, the success of THA is multifactorial. Regardless of which approach is preferred for THA, it is important to find an optimised compromise amongst hip biomechanics, tribology, and post-surgery functionality. Orientation of the prosthetic components is one of the critical factors during THA in order to achieve stable joint and ideal range of motion so that the patient could accomplish their activities of daily living. Intraoperative radiograph using digital image intensifier and/or the use of navigation or robotic systems is recommended to verify correct trial implant positioning and to assure correct offset and leg length. Precise preoperative templating remains an essential for prerequisite for an accurate reconstruction of the hip joint. In this overall concept, MIS THA plays an important role in order to maintain this procedure as one of the most successful procedures in orthopaedic surgery.

\section{Declaration}

Acknowledgments: All authors made contributions to the interpretation of data and have been involved in drafting the manuscript. All authors read and approved the final manuscript.

Informed consent was obtained from all individual participants included in the study.

Availability of data and materials: All data could be requested in the University Hospital of Regensburg (Department of Orthopedics), at any time.

\section{References}

1. Rachbauer F. Minimally invasive total hip arthroplasty. Anterior approach Orthopade. 2006; 35: 723-724, 726-729.

2. Röttinger H. Minimally invasive anterolateral approach for total hip replacement (OCM technique). Oper Orthop Traumatol. 2010; 22: 421-430.

3. Sander K, Layher F, Babisch J, et al. Evaluation of results after total hip replacement using a minimally invasive and a conventional approach. Clinical scores and gait analysis. Z Orthop Unfall. 2011; 149: 191-199.

4. Cheng $\mathrm{T}$, Feng JG, Liu T, et al. Minimally invasive total hip arthroplasty: a systematic review. Int Orthop. 2009; 33: 1473-1481.

5. Goosen JH, Kollen BJ, Castelein RM, et al. Minimally invasive versus classic procedures in total hip arthroplasty: a double-blind randomized controlled trial. Clin Orthop Relat Res. 2011; 469: 200-208.

6. Mazoochian F, Weber P, Schramm S, et al. Minimally invasive total hip arthroplasty: a randomized controlled prospective trial. Arch Orthop Trauma Surg. 2009; 129: 1633-1639.

7. Sendtner E, Borowiak K, Schuster T, et al. Tackling the learning curve: comparison between the anterior, minimally invasive (Micro-hip ${ }^{\circledR}$ ) and the lateral, transgluteal (Bauer) approach for primary total hip replacement. Arch Orthop Trauma Surg. 2010; 131: 597-602.

8. Wohlrab D, Droege JW, Mendel T, et al. Minimally invasive vs. transgluteal total hip replacement. A 3-month follow-up of a prospective randomized clinical study. Orthopade. 2008; 37: 1121-1126.

9. Yang C, Zhu Q, Han Y, et al. Minimally-invasive total hip arthroplasty will improve early postoperative outcomes: a prospective, randomized, controlled trial. Ir J Med Sci. 2010; 179: 285-290.

10. Matziolis D, Wassilew G, Strube $P$, et al. Differences in muscle trauma quantifiable in the laboratory between the minimally invasive anterolateral and transgluteal approach. Arch Orthop Trauma Surg. 2011; 131: 651-655.

11. Müller M, Tohtz S, Springer I, et al. Randomized controlled trial of abductor muscle damage in relation to the surgical approach for primary total hip replacement: minimally invasive anterolateral versus modified direct lateral approach. Arch Orthop Trauma Surg. 2011; 131: 179-189.

12. Musil D, Stehlík J, Verner M. A comparison of operative invasiveness in minimally invasive anterolateral hip replacement (MIS-AL) and standard hip procedure, using biochemical markers. Acta Chir Orthop Traumatol Cech. 2008; 75: 16-20.

13. Yang $\mathrm{C}$, Zhu $\mathrm{Q}$, Han $\mathrm{Y}$, et al. Minimally-invasive total hip arthroplasty will improve early postoperative outcomes: a prospective, randomized, controlled trial. Ir J Med Sci. 2010; 179: 285-290.

14. Fink B, Mittelstaedt A, Schulz MS, et al. Comparison of a minimally invasive posterior approach and the standard posterior approach for total hip arthroplasty. A prospective and comparative study. J Orthop Surg Res. 2010; 5: 46.

15. Martin R, Clayson PE, Troussel S, et al. Anterolateral minimally invasive total hip arthroplasty a prospective randomized controlled study with a follow-up of 1 year. J Arthroplasty. 2011; 26: 1362-1372.

16. Sendtner E, Borowiak K, Schuster T, et al. Tackling the learning curve: comparison between the anterior, minimally invasive (Micro-hip ${ }^{\circledR}$ ) and the lateral, transgluteal (Bauer) approach for primary total hip replacement. Arch Orthop Trauma Surg. 2010; 131: 597-602.

17. Vicente JR, Croci AT, Camargo OP. Blood loss in the minimally invasive posterior approach to total hip arthroplasty: a comparative study. Clinics (Sao Paulo). 2008; 63: 351-356.

18. Yang C, Zhu Q, Han Y, et al. Minimally-invasive total hip arthroplasty will improve early postoperative outcomes: a prospective, randomized, controlled trial. Ir J Med Sci. 2010; 179: 285-290.

19. Fink B, Mittelstaedt A, Schulz MS, et al. Comparison of a minimally invasive posterior approach and the standard posterior approach for total hip arthroplasty. A prospective and comparative study. J Orthop Surg Res. 2010; 5: 46 .

20. Sander K, Layher F, Babisch J, et al. Evaluation of results after total hip replacement using a minimally invasive and a conventional approach. Clinical scores and gait analysis. 2011; 149: 191-199.

21. Varela Egocheaga JR, Suárez-Suárez MÁ, Fernández-Villán M, et al. Minimally invasive posterior approach in total hip arthroplasty. Prospective randomised trial. An Sist Sanit Navar. 2010; 33: 133-143.

22. Duwelius PJ, Moller HS, Burkhart RL, et al. The economic impact of minimally invasive total hip arthroplasty. J Arthroplasty. 2011; 26: 883-885.

23. Bernasek TL, Lee WS, Lee $\mathrm{HJ}$, et al. Minimally invasive primary THA: anterolateral intermuscular approach versus lateral transmuscular approach. Arch Orthop Trauma Surg. 2010; 130: 1349-1354.

24. Mouilhade F, Matsoukis J, Oger $\mathrm{P}$, et al. Component positioning in primary total hip replacement: a prospective comparative study of two anterolateral approaches, minimally invasive versus gluteus medius hemimyotomy. Orthop Traumatol Surg Res. 2011; 97: 14-21.

25. Chow J, Penenberg B, Murphy S. Modified micro-superior percutaneouslyassisted total hip: early experiences \& case reports. Curr Rev Musculoskelet Med. 2011; 4: 146-150.

26. Hueter C. Grundriss der Chirurgie. Leipzig (G): FCW Vogel. 1883: 129-200. German.

27. Smith-Petersen MN, Larson CB, et al. Complications of old fractures of the neckof the femur; results of treatment of vitallium-mold arthroplasty. $\mathrm{J}$ Bone Joint Surg Am. 1947; 29: 41-48.

28. Judet J, Judet H. Anterior approach in total hip arthroplasty. Presse Med. 1985; 14: 1031-1033.

29. Michel MC, Witschger P. Micro Hip: A minimally invasive procedure for total hip replacement surgery using a modified Smith-Peterson approach. Ortop Traumatol Rehabil. 2007; 9: 46-51. 
30. Sendtner E, Borowiak K, Schuster T, et al. Tackling the learning curve: comparison between the anterior, minimally invasive (Micro-hip ${ }^{\circledR}$ ) and the lateral, transgluteal (Bauer) approach for primary total hip replacement. Arch Orthop Trauma Surg. 2010; 131: 597-602.

31. Sendtner E, Borowiak K, Schuster T, et al. Tackling the learning curve: comparison between the anterior, minimally invasive (Micro-hip ${ }^{\circledR}$ ) and the lateral, transgluteal (Bauer) approach for primary total hip replacement. Arch Orthop Trauma Surg. 2010; 131: 597-602

32. McFarland B, Osborne G. Approach to the hip: A suggested improvement on Kocher's method. J Bone Joint Surg Br. 1954; 36B: 364-367.

33. Hardinge K. The direct lateral approach to the hip. J Bone Joint Surg. 1982; 64: 17-19.

34. Bauer R, Kerschbaumer F, Poisel S, et al. The transgluteal approach to the hip joint. Arch Orthop Trauma Surg. 1979; 95: 47-49.

35. Moore AT. Metal hip joint; a new self-locking vitallium prosthesis. South Med J. 1952; 45: 1015-1019.

36. Kocher T. Textbook of Operative Surgery. $3^{\text {rd }}$ English Ed. New York (NY) Toronto, MacMillan. 1911: 33-34.

37. Gänsslen A, Grechenig S, Nerlich M, et al. Standard approaches to the acetabulum part 1: Kocher-Langenbeck approach. Acta Chir Orthop Traumatol Cech. 2016; 83: 141-146.

38. Schmidt-Braekling T, Waldstein W, Akalin E, et al. Minimal invasive posterio total hip arthroplasty: are 6 weeks of hip precautions really necessary? Arch Orthop Trauma Surg. 2015; 135: 271-274

39. Fink B, Mittelstaedt A, Schulz MS, et al. Comparison of a minimally invasive posterior approach and the standard posterior approach for total hip arthroplasty a prospective and comparative study. Journal of Orthopaedic Surgery and Research. 2010; 5: 46

40. Alecci V, Valente $M$, Crucil $M$, et al. Comparison of primary total hip replacements performed with a direct anterior approach versus the standard lateral approach: peri-operative findings. Orthop Traumatol. 2011; 12: 123129.

41. Bala subramaniam U, Dowsey M, Ma F, et al. Functional and clinical outcomes following anterior hip replacement: a 5-year comparative study versus posterior approach. ANZ J Surg. 2016; 86: 589-593.

42. Brismar $\mathrm{BH}$, Hallert $\mathrm{O}$, Tedhamre $\mathrm{A}$, et al. Early gain in pain reduction and hip function, but more complications following the direct anterior minimally invasive approach for total hip arthroplasty: a randomized trial of 100 patients with 5 years of follow up. Acta Orthop. 2018; 89: 484-489.

43. D'Arrigo C, Speranza A, Monaco E, et al. Learning curve in tissue sparing total hip replacement comparison between different approaches. J Orthop Traumatol. 2009; 10: 47-54

44. Dienstknecht $\mathrm{T}$, Lüring $\mathrm{C}$, Tingart $\mathrm{M}$, et al. Total hip arthroplasty through the mini-incision (Micro-hip) approach versus the standard transgluteal (Bauer) approach: a prospective, randomised study. J Orthop Surg (Hong Kong). 2014; 22: 168-172.

45. Fink B, Mittelstaedt A, Schulz MS, et al. Comparison of a minimally invasive posterior approach and the standard posterior approach for total hip arthroplasty. A prospective and comparative study. J Orthop Surg Res. 2010; 5: 46

46. Goosen JH, Kollen BJ, Castelein RM, et al. Minimally invasive versus classic procedures in total hip arthroplasty: a double-blind randomized controlled trial. Clin Orthop Relat Res. 2011; 469: 200-208.

47. Hozack W, Klatt BA. Minimally invasive two-incision total hip arthroplasty: is the second incision necessary? Semin Arthrop. 2008; 19: 205-208.

48. Ilchmann T, Gersbach S, Zwicky L, et al. Standard transgluteal versus minima invasive anterior approach in hip arthroplasty: a prospective, consecutive cohort study. Orthop Rev (Pavia). 2013; 5: 31.

49. Kawarai Y, Lida S, Nakamura J, et al. Does the surgical approach influence the implant alignment in total hip arthroplasty? Comparative study between the direct anterior and the anterolateral approaches in the supine position. Int
Orthop. 2017; 41: 2487-2493.

50. Martin R, Clayson PE, Troussel S, et al. Anterolateral minimally invasive total hip arthroplasty a prospective randomized controlled study with a follow-up of 1 year. J Arthroplasty. 2011; 26: 1362-1372.

51. Más Martínez J, Sanz-Reig J, Morales-Santías M, et al. Comparative cohort study of the Super Path approach and the conventional posterior approach in primary cementless hip replacement surgery. Rev Esp Cir Ortop Traumatol. 2019; 63: 346-354

52. Matziolis D, Wassilew G, Strube $P$, et al. Differences in muscle trauma quantifiable in the laboratory between the minimally invasive anterolateral and transgluteal approach. Arch Orthop Trauma Surg. 2011; 131: 651-655.

53. Mazoochian F, Weber P, Schramm S, et al. Minimally invasive total hip arthroplasty: a randomized controlled prospective trial. Arch Orthop Trauma Surg. 2009; 129: 1633-1639.

54. Mouilhade F, Matsoukis $\mathrm{J}$, Oger $\mathrm{P}$, et al. Component positioning in primary total hip replacement: a prospective comparative study of two anterolateral approaches, minimally invasive versus gluteus medius hemimyotomy. Orthop Traumatol Surg Res. 2011; 97: 14-21.

55. Müller M, Tohtz S, Springer I, et al. Randomized controlled trial of abductor muscle damage in relation to the surgical approach for primary total hip replacement: minimally invasive anterolateral versus modified direct lateral approach. Arch Orthop Trauma Surg. 2011; 131: 179-189.

56. Ouyang C, Wang $\mathrm{H}$, Meng $\mathrm{W}$, et al. Randomized controlled trial of comparison between the Super Path and posterolateral approaches in total hip arthroplasty. Zhongguo Xiu Fu Chong Jian Wai Ke Za Zhi. 2018; 32: 1500-1506.

57. Pogliacomi F, De Filippo M, Paraskevopoulos A, et al. Mini incision direct lateral approach versus anterior mini invasive approach in total hip replacements: Results 1 year after surgery. Acta Biomed. 2012; 83: 114-121.

58. Reichert JC, von Rottkay E, Roth $F$, et al. A prospective randomized comparison of the minimally invasive direct anterior and the transgluteal approach for primary total hip arthroplasty. BMC Musculoskeletal Disorders. 2018; 19: 241

59. Rykov K, Reininga IHF, Sietsma MS, et al. Posterolateral vs Direct Anterior Approach in Total Hip Arthroplasty (POLADA Trial): A Randomized Controlled Trial to Assess Differences in Serum Markers. J Arthroplasty. 2017; 32: 3652 3658.

60. Sendtner E, Borowiak K, Schuster T, et al. Tackling the learning curve: comparison between the anterior, minimally invasive (Micro-hip ${ }^{\circledR}$ ) and the lateral, transgluteal (Bauer) approach for primary total hip replacement. Arch Orthop Trauma Surg. 2010; 131: 597-602.

61. Sirtori P, Peretti GM, Ulivi M, et al. Comparison of early functional outcomes between two different surgical approaches for total hip arthroplasty. J Bio Regul Homeost Agents. 2018; 32: 89-96.

62. Varela Egocheaga JR, Suárez-Suárez MÁ, Fernández-Villán $M$, et al Minimally invasive posterior approach in total hip arthroplasty. Prospective randomised trial. An Sist Sanit Navar. 2010; 33: 133-143.

63. Wayne N, Stoewe R. Primary THA: a comparison of the lateral hardinge approach to an anterior mini-invasive approach. Orthop Rev (Pavia). 2009; 1: 27

64. Wohlrab D, Droege JW, Mendel T, et al. Minimally invasive vs. transgluteal total hip replacement. A 3-month follow-up of a prospective randomized clinical study. Orthopade. 2008; 37: 1121-1126.

65. Xie J, Zhang H, Wang L, et al. Comparison of supercapsular percutaneously assisted approach total hip versus conventional posterior approach for total hip arthroplasty: a prospective, randomized controlled trial. Journal of Orthopaedic Surgery and Research. 2017; 12: 138.

66. Yan T, Tian S, Wang Y, et al. Comparison of early effectiveness between Super Path approach and Hardinge approach in total hip arthroplasty. Zhongguo Xiu Fu Chong Jian Wai Ke Za Zhi. 2017; 31: 17-24.

67. Yang C, Zhu Q, Han Y, et al. Minimally-invasive total hip arthroplasty will 
improve early postoperative outcomes: a prospective, randomized, controlled trial. Ir J Med Sci. 2010; 179: 285-290.

68. Yuan H, Zhu J, Sun Z, et al. Comparison of effectiveness between Super Path approach and posterolateral approach in total hip arthroplasty. Zhongguo Xiu Fu Chong Jian Wai Ke Za Zhi. 2018; 32: 14-19.

69. Zhao M, Hu Y, Zeng M, et al. Short-term effectiveness of minimally invasive total hip arthroplasty by direct anterior approach. Zhongguo Xiu Fu Chong Jian Wai Ke Za Zhi. 2017; 31: 11-16.

70. Müller M, Tohtz S, Springer I, et al. Randomized controlled trial of abductor muscle damage in relation to the surgical approach for primary total hip replacement: minimally invasive anterolateral versus modified direct lateral approach. Arch Orthop Trauma Surg. 2011; 131: 179-189.

71. Kubes J, Landor I, Podskubka A, et al. Total hip replacement from a MIS AL approach (comparison with a standard anterolateral approach). Acta Chir Orthop Traumatol Cech. 2009; 76: 288-294.

72. Renkawitz T, Tingart M, Grifka J, et al. Computer-assisted total hip arthroplasty: coding the next generation of navigation systems for orthopedic surgery. Expert Rev Med Devices. 2009; 6: 507-514

73. Sendtner E, Borowiak K, Schuster T, et al. Tackling the learning curve: comparison between the anterior, minimally invasive (Micro-hip ${ }^{\circledR}$ ) and the lateral, transgluteal (Bauer) approach for primary total hip replacement. Arch Orthop Trauma Surg. 2010; 131: 597-602.

74. Dienstknecht $\mathrm{T}$, Lüring $\mathrm{C}$, Tingart $\mathrm{M}$, et al. Total hip arthroplasty through the mini-incision (Micro-hip) approach versus the standard transgluteal (Bauer) approach: a prospective, randomised study. J Orthop Surg (Hong Kong). 2014; 22 : 168-172

75. Jerosch J. Minimally Invasive Hip Replacement. Dtsch Arztebl. 2006; 103: A-3333.
76. Mjaaland KE, Svenningsen S, Fenstad AM, et al. Implant Survival After Minimally Invasive Anterior or Anterolateral vs. Conventional Posterior or Direct Lateral Approach: An Analysis of 21,860 Total Hip Arthroplasties from the Norwegian Arthroplasty Register (2008 to 2013). J Bone Joint Surg Am. 2017; 99: 840-847.

77. Kawarai Y, Lida S, Nakamura J, et al. Does the surgical approach influence the implant alignment in total hip arthroplasty? Comparative study between the direct anterior and the anterolateral approaches in the supine position. Int Orthop. 2017; 41: 2487-2493.

78. Goulding K, Beaulé PE, Kim PR, et al. Incidence of Lateral Femoral Cutaneous Nerve Neuropraxia after Anterior Approach Hip Arthroplasty. Clin Orthop Relat Res. 2010; 468: 2397-2404.

79. Duwelius PJ, Moller HS, Burkhart RL, et al. The economic impact of minimally invasive total hip arthroplasty. J Arthroplasty. 2011; 26: 883-885

80. Mahmood A, Zafar MS, Majid I, et al. Minimally invasive hip arthroplasty: a quantitative review of the literature. Br Med Bull. 2007; 84: 37-48.

81. Varela Egocheaga JR, Suárez-Suárez MÁ, Fernández-Villán $M$, et al. Minimally invasive posterior approach in total hip arthroplasty. Prospective randomised trial. An Sist Sanit Navar. 2010; 33: 133-143.

82. Kehlet $\mathrm{H}$. History and future challenges in fast-track hip and knee arthroplasty. Orthopade. 2020; 49: 290-292.

83. Lloyd JM, Wainwright T, Middleton RG. What is the role of minimally invasive surgery in a fast track hip and knee replacement pathway? The Royal College of Surgeons of England. 2012; 94: 148-151.

84. Luna IE, H Kehlet H, B Peterson B, et al. Early patient-reported outcomes versus objective function after total hip and knee arthroplasty: a prospective cohort study. Bone Joint J. 2017; 99-B: 1167-1175. 